\title{
Development of Frequency Modulated Array Antennas for Millimeter-Wave Communications
}

\author{
Shaddrack Yaw Nusenu $\mathbb{D}^{1,2}$ \\ ${ }^{1}$ University of Electronic Science and Technology of China (UESTC), China \\ ${ }^{2}$ Koforidua Technical University (KTU), Ghana \\ Correspondence should be addressed to Shaddrack Yaw Nusenu; nusenu2012gh@yahoo.com
}

Received 24 December 2018; Revised 15 March 2019; Accepted 7 April 2019; Published 16 April 2019

Academic Editor: Daniele Pinchera

Copyright (C) 2019 Shaddrack Yaw Nusenu. This is an open access article distributed under the Creative Commons Attribution License, which permits unrestricted use, distribution, and reproduction in any medium, provided the original work is properly cited.

\begin{abstract}
With the massive growth of wireless data in mobile broadband communications, millimeter-wave (mm-wave) communication is an alternative enabling technique for fifth generation (5G) wireless communication systems. More importantly, mm-wave offers large frequency spectrum bands ranging from $30 \mathrm{GHz}$ to $300 \mathrm{GHz}$ that can be utilized to provide very high capacity (i.e., multigigabits per-second data rates). Moreover, because of the small wavelength at mm-wave frequencies, we can exploit large antenna elements in a small physical area, meaning beamforming schemes are feasible. Nevertheless, high directional antennas should be used due to overcoming the severe path loss and absorption in mm-wave frequencies. Further, the antennas should be steerable in angle and range directions to support point-to-point (multipoint) communications. So far, mm-wave communication has utilized phased-array antennas arrangement which is solely angle dependent. This review paper presents recent array technology, namely, frequency modulated frequency diverse array (FDA) for mm-wave communication applications with an emphasis on beamforming. In FDA, small frequency increment is added across the elements. In doing so, an array beam is generated which is angle-range-time dependent without the need of phase shifters. This feature has several promising potentials in $\mathrm{mm}$-wave communications. In this review, the object is to bring to the fore this advance FDA technology to mm-wave communications community to call for more investigations. We review FDA research progress up to date and highlight the potential applications in mm-wave communications.
\end{abstract}

\section{Introduction}

Due to massive growth of wireless data in mobile broadband communications, millimeter-wave (mm-wave) communication is put forward as the most attractive solution for $5 \mathrm{G}$ technology [1-5]. Because mm-wave communications frequency ranges from $30 \mathrm{GHz}$ to $300 \mathrm{GHz}$, higher data rates can be achieved especially for broadband wireless communications. This large bandwidth has also solved the scarcity problem of frequency spectrum. Besides, the small wavelength of $\mathrm{mm}$ wave signals has inspired multiple-input multiple-output (MIMO) techniques. And also more array elements can be deployed in a physically limited space $[6,7]$ to provide increase beamforming gain and spectral efficiency $[1,8-12]$.

Beamforming schemes in mm-wave communications can be exploited to overcome the path loss at $\mathrm{mm}$-wave frequencies. The mm-wave channel characteristics can influence the choice of physical layer and medium access control layer schemes, as well as the designing of the hardware [1]. From this background, multiple antenna technology is crucial in mm-wave communications as it can increase the channel capacity. The transceiver can exploit multiple antennas to derive the following advantages: multiplexing and diversity gain or antenna beam/gain [1]. Furthermore, directional steerable antenna configurations in range and angle dependent can significantly improve the received signal-to-interference-to-noise ratio (SINR) [1] .

Phased-array antennas have been the most common antennas arrangement widely used in many applications such as wireless communications, radar systems, and others [1621]. Phased-array antennas have high directivity because they can steer the beam electronically. This feature is essential in communications, especially for detecting/tracking mobile users and suppressing sidelobe regions [17]. However, some of its limitations are listed as follows: (1) high cost of electronic phase shifters [18, 19], (2) fixed angle directions for all 


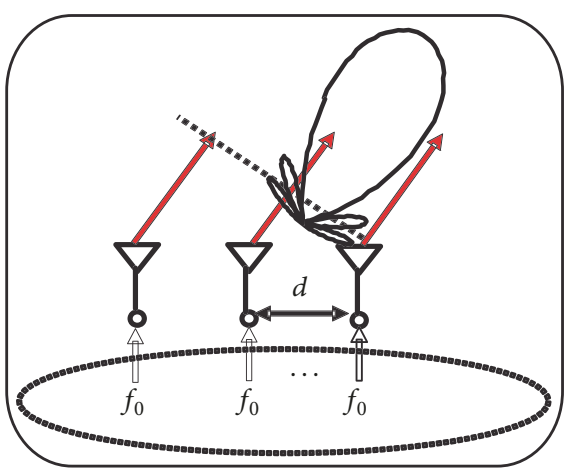

(a)

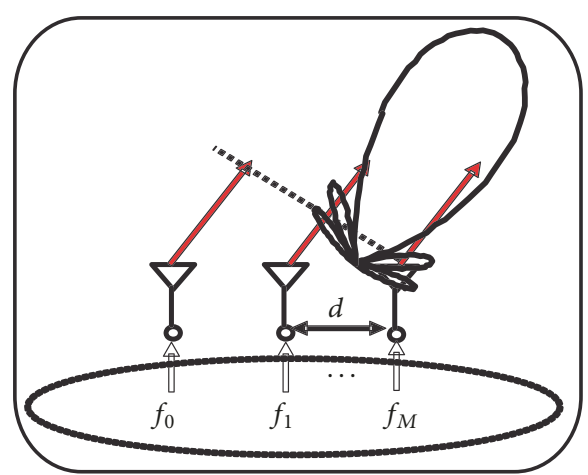

(b)

FIGURE 1: Comparisons of array geometry: (a) conventional phased-array, (b) FDA antenna.

ranges (this implies that phased-array provides range-time independent transmit beampattern), (3) range-dependent suppression is not feasible in phased-array, (4) for instance, let us assume the case for two mobile users located at the same angle direction but distinct ranges, using phased-array antenna configuration. Its beam steering will illuminate these users in one angle but for all the ranges. Consequently, this will degrade the received SINR of the mobile users.

In recent years, a new beam technology was proposed, namely, frequency diverse array (FDA) by $[22,23]$ to handle the challenge facing phased-array antenna arrangement. In FDA, a small frequency increment is added across the elements which result in angle-range-time beamforming [22]. Unlike the conventional phased-array, FDA enables beam scan without phase shifters with additional degree of freedom [23]. Though, FDA technology may be a variant of multipleinput multiple-output (MIMO) [24] systems, but both systems present distinct configurations: FDA transmitted signals are closely overlapped in frequencies, while MIMO can employs orthogonal signals or nonorthogonal signals from either closely or widely separated antennas [25, 26]. Likewise, FDA functionality is also different from orthogonal frequency division multiplexing (OFDM) [27]. The latter uses orthogonal subcarriers [28]. Moreover, frequency scanning arrays [29] and FDA have different functionalities. The each former employed element at a given instant of time uses the same frequency [30]. In [31], it was pointed out that FDA beampattern generates a global maximum at different angle and ranges locations. This property can be utilized for detecting multiple mobile users. More importantly, due to the frequency increments, the apparent scan angle is different from its nominal scan angle and the actual beam steering direction is difficult to be effectively predicted unlike the phased-arrays [32]. Another unique property of FDA is that the phases of the transmitted signal can be formed constructively or destructively in certain regions. This feature is also attractive for interference suppression [32] as in mm-wave communications high directional antennas are required. The authors in [33-36] discussed the applications of FDA in radar. Also, FDA antennas have been utilized in wireless communications for security applications [27, 37-40] and very recently, FDA antennas in wireless applications were presented in $[15,41]$. Again, in the application of codesign radar and communication (RadCom), FDA has been very attractive [42-46].

This paper presents a review on recent proposed FDA antenna technology. Specifically, we highlight the potential applications of employing FDA in mm-wave communications and also appeal to wireless communication communities to call for more investigations on FDA antenna development especially in $\mathrm{mm}$-wave $5 \mathrm{G}$ communications and even nonorthogonal multiple access (NOMA) schemes. In addition, we present some array configurations suitable for mm-wave communications for consideration.

The rest of this paper is organized as follows: Section 2 presents an overview and some necessary details about frequency diverse array (FDA) antenna (narrowband and ultrawideband). Section 3 explains why FDA is suitable for mm-wave transmissions. Section 4 presents a hybrid Butler matrix FDA architecture for mm-wave communications. Section 5 presents discussions and future possible directions of FDA. Finally, we draw conclusion in Section 6.

\section{Mathematical Formulation of FDA Antenna}

\subsection{Narrow-Band FDA Antenna Formulation}

(a) FDA Formulation. Figure 1 shows array geometry comparisons between phased-array and FDA antennas. Without loss of generality, in this paper, identical transmitted signals are assumed with a frequency increment $\Delta f$ for each element and element spacing is denoted as $d$ as depicted in Figure 1.

From Figure 1(b), the radiation frequency considering the $m$ th element is [32]

$$
f_{m}=f_{0}+(m-1) \cdot \Delta f \quad m=1,2,3, \ldots, M
$$

where $f_{0}$ is defined as the carrier frequency. Note that the frequency increment $\Delta f$ can be uniformly and nonuniformly designed.

According to $[15,32]$, and using the first element as reference point, FDA beampattern steering vector is

$$
\mathbf{a}(\theta, r, t)=\mathbf{a}_{\theta}(\theta) \odot \mathbf{a}_{r}(r) \odot \mathbf{a}_{t}(t)
$$




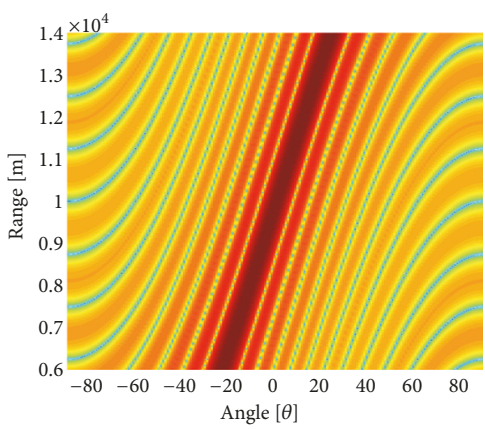

(a)

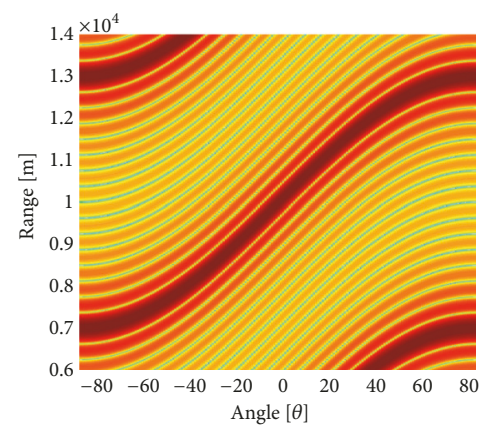

(b)

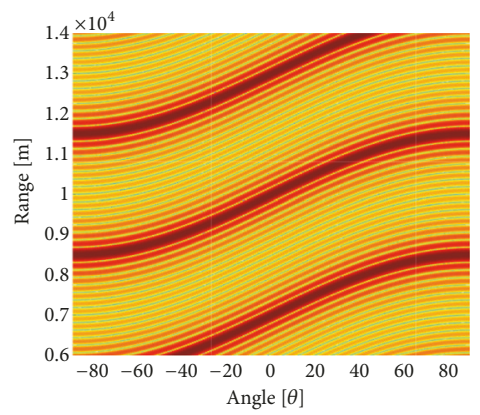

(c)

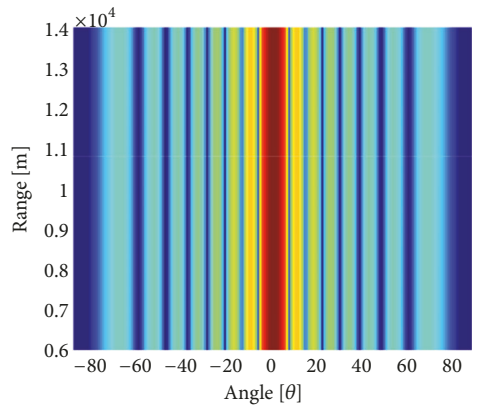

(d)

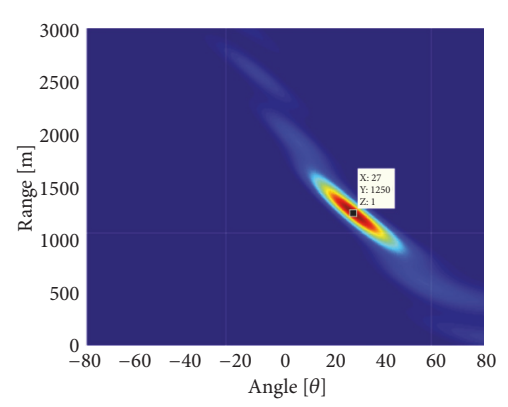

(e)

FiguRE 2: FDA beamforming comparison using distinct $\Delta f$ (a-c): (a) $\Delta f=15 \mathrm{kHz}$, (b) $\Delta f=50 \mathrm{kHz}$, (c) $\Delta f=100 \mathrm{kHz}$, (d) shows PA for comparison purposes $\Delta f=0: M=16, d=0.5 \lambda$ and $f_{0}=30 \mathrm{GHz}$; (e) FDA beamforming in range-angle focusing using log-frequency increment for $\mathrm{mm}$-wave systems to support a few hundred meters to $\mathrm{km}$ distance transmission.

where

$$
\begin{aligned}
& \mathbf{a}_{\theta}(\theta)=\left[1, \exp \left(-j \xi_{1}\right), \cdots, \exp \left(-j \xi_{1} M\right)\right]^{T} \\
& \mathbf{a}_{r}(r)=\left[1, \exp \left(-j \xi_{2}\right), \cdots, \exp \left(-j \xi_{2} M\right)\right]^{T} \\
& \mathbf{a}_{t}(t)=\left[1, \exp \left(-j \xi_{3}\right), \cdots, \exp \left(-j M \xi_{3}\right)\right]^{T}
\end{aligned}
$$

where $\xi_{1}=2 \pi m d \sin \theta / \lambda, \xi_{2}=2 \pi \Delta f r / c, \xi_{3}=2 \pi \Delta f t, \odot$ and $[\cdot]^{T}$ denotes Hadamard operator and transpose operator, respectively, $c$ is defined as the light speed, $r$ denotes the distance, and note that $r_{m} \approx r-(m-1) d \sin \theta$. For comparison purposes, the beampattern steering vector of phased-array is

$$
\mathbf{a}^{\text {phased-array }}(\theta)=\left[1, \exp \left(-j \xi_{1}\right), \cdots, \exp \left(-j \xi_{1} M\right)\right]^{T}
$$

The array factor of FDA antenna can be given as

$$
A F(\theta, r, t)=\sum_{m=0}^{M-1} w_{m}^{*} \exp \left(j \Upsilon_{F D A}\right)
$$

where $w_{m}$ denote the transmit weight, $\Upsilon_{F D A}=2 \pi(m \Delta f(t-$ $\left.r / c)[t-(r-d m \sin \theta) / c]+f_{0} d m \sin \theta / c\right)$. The term $m 2 \pi \Delta f d \sin \theta / c$ is ignored in $\Upsilon_{F D A}$ since $r \gg(M-1) d \sin \theta$ and $f_{0} \gg \Delta f$, and ${ }^{*}$ being the conjugate operator.

The following remarks about FDA antennas based on above expressions are in order [32, 34].

(1) When $\Delta f$ is fixed, we get range $r$ dependent beam scanning.
(2) When $r$ is fixed, we also have $\Delta f$ beam dependent.

(3) If ( $\Delta f=0)$ is considered, FDA is just phased-array antenna.

(4) The higher the value of $\Delta f$, the better the beamwidth resolution.

In this review paper, we highlighted the potentials of employing frequency modulated array (frequency diverse array (FDA)) beamforming rather than the conventional phased-array antennas (PA) in millimeter-wave (mm-wave) applications. The FDA has the capability of providing directional beam gain in angle-range-time focusing dependent due to the tiny (small) frequency increment across each element index. The frequency increment $\Delta f$ is far smaller than the carrier frequency (i.e., $f_{0} \gg \Delta f$ ) in practice. It should be noted that $\Delta f$ induces the FDA beamforming with more degrees of freedom. In addition, the range interference can be significantly suppressed using FDA which is not accessible to PA. In Figure 2, we have simulated FDA beamforming with distinct values of $\Delta f$ at carrier frequency $f_{0}=30 \mathrm{GHz}$.

As shown in Figure 2, we can make some remarks.

(1) The $\Delta f$ controls the range-angle focusing dependent and spatial distribution of the FDA transmit beamforming during the signal propagation into free space.

(2) It was observed that as the carrier frequency becomes higher (i.e., as in the case of mm-wave applications), $\Delta f$ should be high enough to provide good spatial 
TABLE 1: FDA versus phased array [15].

\begin{tabular}{lcccc}
\hline $\begin{array}{l}\text { Antenna } \\
\text { array }\end{array}$ & Transmitted signals & Coherent gain (array) & Transmit capabilities & $\begin{array}{c}\text { Range-dependent } \\
\text { suppression }\end{array}$ \\
\hline FDA & coherent & yes & $\begin{array}{c}\text { angle-range-time } \\
\text { dependent }\end{array}$ & yes \\
Phased array & coherent & yes & angle dependent only & no \\
\hline
\end{tabular}

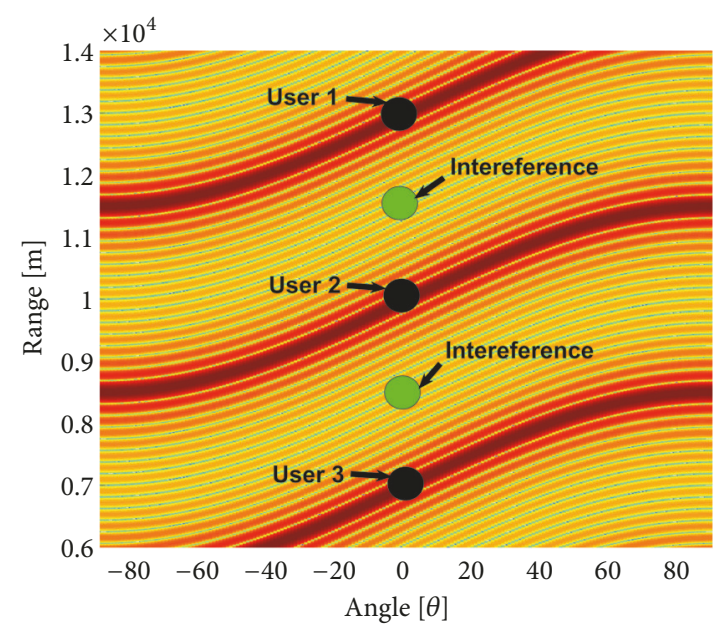

FIGURE 3: Range-dependent suppression in FDA transmit beamforming by tuning $\Delta f=100 \mathrm{kHz}$.

resolution and improve directionality; see Figure 2(c). Additionally, $\Delta f$ is not the whole bandwidth.

(3) Note that matching filter can be employed at the receiver to handle $\Delta f$.

(4) In fact, $\Delta f$ renders some transmit bandwidth expansion. Hence, a compromise should be created between bandwidth expansion and performance improvement.

More importantly, in Figure 2(e), we have simulated FDA beamforming considering a few $\mathrm{km}$ distance and angle $\left(27^{\circ}, 1250 m\right)$ to illustrate the short distance mm-wave communications. In this beamforming, we consider the following parameters: range $(0 \mathrm{~km} \sim 3 \mathrm{~km})$, angle $\left(-80^{\circ} \sim 80^{\circ}\right)$, and log-frequency increment given as $\Delta f_{m}=\log (m+1) \Delta f$ and $\Delta f=400 \mathrm{kHz} \ll f_{0}=30 \mathrm{GHz}$. From the figure, it can be noticed that adopting log-frequency increment, we can decouple the range-angle dependent to focus the beamforming at specific location for $\mathrm{mm}$-wave systems to support a few hundred $/ k$-meters distance for access network. Note that the electromagnetic wave will arrived at such location at time $t=0.0042 \mathrm{~ms}$.

Actually, FDA beamforming is feasible for $\mathrm{mm}$-wave applications. For instance, channels under mm-wave are correlated not independent and identically distributed (i.i.d). Hence in physical layer security in mm-wave where the desired receiver and undesired receiver are highly correlated (i.e., close-located), FDA is more feasible than PA due to the angle-range focusing dependent. Secondly, as mentioned before, we can suppress range-dependent interferences by choosing appropriate $\Delta f$; see Figure 3.
Furthermore, Table 1 compares the FDA antenna and phased-array antenna in terms of transmission signals, coherent gain, and transmitting abilities. And Table 2 compares different FDA antenna configuration.

2.2. Ultrawideband FDA Formulation. Very recently, ultrawideband (UWB) FDA has been reported in [13]. We think that for designing UWB FDA schemes for mm-wave communication systems, many areas of applications could benefit from such system. Moreover, UWB has been employed in radar [47-49] and wireless communication fields [50] due to their promising advantages.

Analogous to [13], we illustrate continuous FDA waveform and pulsed FDA waveform in Figure 4. $t_{s}$ is defined as the start time and $t_{e}$ denotes the ending time. The pulse duration is $T_{p}=t_{e}-t_{s}$. For continuous FDA waveform shown in the upper subplot, the start time is $t_{s}=0$ and $t_{e}$ is infinite. Suppose the transmitted baseband signal is given as $\phi(t)$ with unit energy, i.e., $\int_{T_{p}} \phi(t) \phi^{*}(t) d t=1$, with ${ }^{*}$ being the conjugate operator. And $1 / \Delta f$ is equal to pulse repetition frequency (PRF). Also it is worth mentioning that for a wideband pulsed (WB) FDA, this relation, i.e., $\phi(t-(r-$ $m d \sin \theta) / c) \approx \phi(t-r / c)$, does not hold [13]. Therefore, we can write WB FDA transmit beampattern as [13]

$$
\begin{aligned}
& A F_{W B-F D A}(\theta, r, t) \\
& \quad=\left|\sum_{m=0}^{M-1} w_{m}^{*} \phi\left(t-\frac{r-m d \sin \theta}{c}\right) \exp j m \Upsilon_{F D A}\right|
\end{aligned}
$$


TABLE 2: FDA antenna characteristics [15].

\begin{tabular}{lccccc}
\hline $\begin{array}{l}\text { Antenna } \\
\text { configuration }\end{array}$ & $\begin{array}{c}\text { frequency } \\
\text { increment }\end{array}$ & $\begin{array}{c}\text { Range and } \\
\text { Angle }\end{array}$ & $\begin{array}{c}\text { Computation } \\
\text { complexity }\end{array}$ & $\begin{array}{c}\text { Interference } \\
\text { suppression }\end{array}$ & $\begin{array}{c}\text { User } \\
\text { detection } \\
\text { capability }\end{array}$ \\
\hline $\begin{array}{l}\text { Standard } \\
\text { FDA }\end{array}$ & linear & $\begin{array}{c}\text { Coupled } \\
\text { beampattern } \\
\text { Modified }\end{array}$ & reasonable & good & good \\
FDA & non-linear & $\begin{array}{c}\text { decoupled } \\
\text { beampattern }\end{array}$ & quiet high & better & better \\
\hline
\end{tabular}
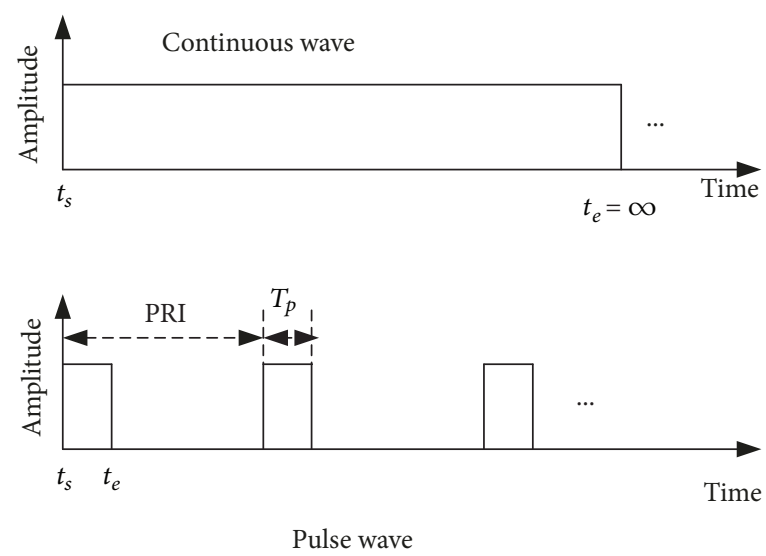

FIgURE 4: Comparisons of continuous FDA waveform and pulsed FDA waveform.

In this paper, we consider rectangular pulse $\phi(t)$ which is given as

$$
\phi(t)= \begin{cases}1, & 0 \leq t_{s} \leq t \leq t_{e}<\frac{1}{\Delta f} \\ 0, & \text { otherwise }\end{cases}
$$

Hence, without loss of generality, the UWB pulsed FDA can be reformulated as

$$
\begin{aligned}
& A F_{U W B-F D A}(\theta, r, t) \\
& = \begin{cases}\left|\sum_{m=0}^{M-1} \exp j m \Upsilon_{F D A}\right|, & t_{s}+\frac{r}{c} \leq t \leq t_{e}+\frac{r}{c} \\
0, & 0 \leq t<\frac{r}{c}+t_{s} \text { or } t>t_{e}+\frac{r}{c}\end{cases}
\end{aligned}
$$

where for sake of simplicity, we assume $w=\left[\begin{array}{llll}1 & 1 & \cdots & 1\end{array}\right]^{T}$. As shown in (14), it can be observed that both continuouswave/narrowband FDA and UWB pulse FDA create anglerange-dependent beampattern. This attribute offers potential applications in $\mathrm{mm}$-wave communications.

In Figure 5, we illustrate the beampattern comparisons between the FDA continuous-wave and FDA pulsed-wave when $T_{p}=1 / 5 \cdot \Delta f$. As seen in Figure 5(a), the FDA continuous-wave beampattern has a scanning periodicity of $1 / \Delta f$, while in Figure $5(\mathrm{~b})$, it can be noticed that at $t_{s}=1 \mathrm{~ms}$, the scanning beam is formed at the distance of $1.4 \mathrm{~ms}$ with a delay. Furthermore, in Figure 6, we compare the transmit beampattern of FDA continuous-wave and FDA pulsed-wave when $t_{0}=0.5 m s$, i.e., $t_{s}<t_{e}<t_{0}$ and $T_{p}=1 / 150 \cdot \Delta f$ is considered. As noticed from the figure, at a particular instant of time, distinct beampatterns can be generated with different angle-range locations.

\section{Feasibility of FDA Antennas for Millimeter-Wave Communications}

3.1. FDA Periodicity in Angle, Range, and Time Factors. According to [32], the maximum field is

$$
\begin{aligned}
\Delta f\left(t-\frac{r}{c}+d \sin \frac{\theta}{\lambda \Delta f}+\frac{d \sin \theta}{c}\right)= & \ell, \\
& \ell=0, \pm 1, \pm 2, \ldots .
\end{aligned}
$$

As shown in (11), we can compute for periodicity factors, namely, time $t$, range $r$, and angle $\theta$, respectively. Again, inspecting (11), FDA antenna arrangement has auto-scanning property [51] which could be exploited at the base station (BS) for $\mathrm{mm}$-wave communication applications as demonstrated in Figure 7.

\subsection{Decoupled Range and Angle Characteristics for Millimeter-} Wave Communications. As already stated in the previous sections, the standard FDA provides coupled angle-rangedependent beampattern which limits its estimation at the FDA peak output. Herein, we demonstrate the capability of decoupling angle-range-dependent FDA beampattern by considering two communication receivers/users located at the same direction but different ranges. Note that in the literature, several authors have proposed different techniques to decouple angle-range dependent of FDA [32, 40, 52-54]. 


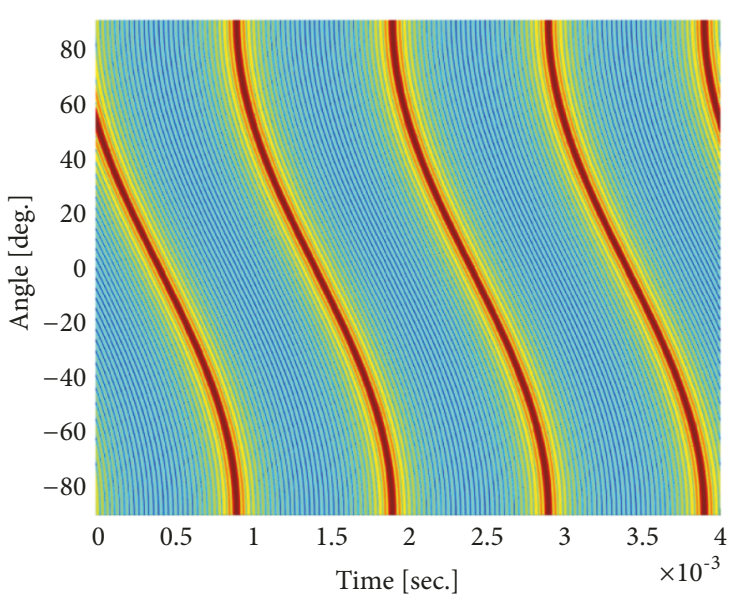

(a)

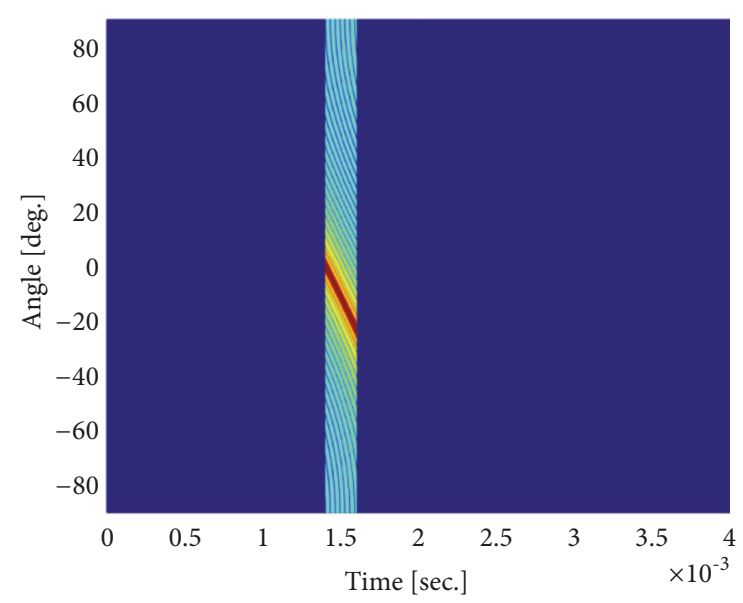

(b)

FIGURE 5: Comparisons of transmit beampatterns: (a) FDA using continuous-wave, (b) FDA pulsed-wave, parameters: $M=32, \Delta f=1 \mathrm{kHz}$, $r=120 \mathrm{~km}$, and $t_{s}=1 \mathrm{~ms}[13]$.

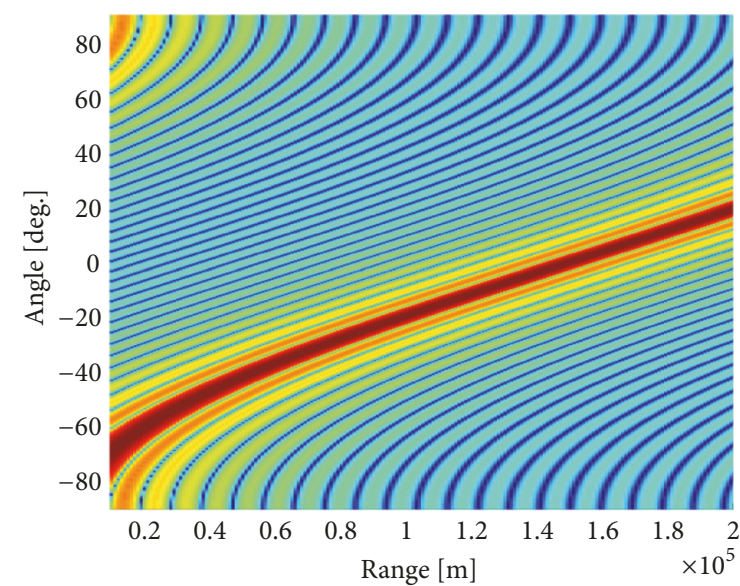

(a)

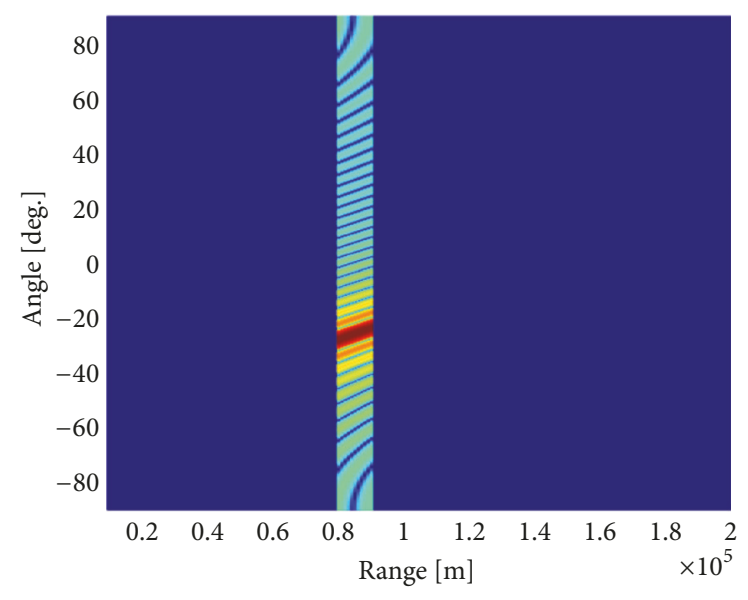

(b)

FIGURE 6: Comparisons of transmit beampatterns: (a) FDA using continuous-wave, (b) FDA pulsed-wave, parameters: $M=32, \Delta f=1 \mathrm{kHz}$, and $t_{s}=0.2 m s[13]$.

Employing (1), we can design nonlinear Chebyshev window-based frequency increment $\Delta f_{m}$ which has the ability to suppress sidelobe regions significantly which is given as

$$
\Delta f_{m}=\Delta f\left\{\frac{\cos \left(M \cos ^{-1}[\vartheta \cos (m \pi / M)]\right)}{\cosh \left(M \cosh ^{-1}(\vartheta)\right)}\right\}
$$

where $\mathcal{Y}=\cosh \left[(M)^{-1} \cosh ^{-1}\left(10^{\varepsilon}\right)\right]$ and $\varepsilon$ is used to control the sidelobe levels. Plugging (12) into (7) and assuming $\mathrm{t}=0$ for the sake of simplicity, we plot the decoupling FDA beampattern in Figure 8. We also illustrated the phased-array beampattern considering two communication receivers/users located at the same direction but different ranges. From Figure 8, it is so apparent that using FDA, we can be able to differentiate between two communication receivers located at $\left(25^{\circ}, 750 \mathrm{~km}\right)$ and $\left(25^{\circ}, 300 \mathrm{~km}\right)$, respectively, than its counterpart phased-array. Therefore, employing FDA antennas at the BS (see Figure 7) can indeed improve the received SINR than phased-array. Additionally, the FDA has better interference suppression capability.

\subsection{FDA Low Probability of Interception (LPI) Studies.}

Wireless communication systems are the most attractive systems for sharing of information between several users due to the nature of propagation channel. On the other hand, information protection is also important; otherwise, the intended receiver information can be compromised. Therefore, security issues are essential in wireless communication systems. Recently, physical layer security has been widely investigated in the literature due to its degree of freedom as compared to the traditional cryptography keys sharing methods [55-57]. Apparently, physical layer security exploits the wireless channels to transmit secure information to the intended receiver without being intercepted by the 


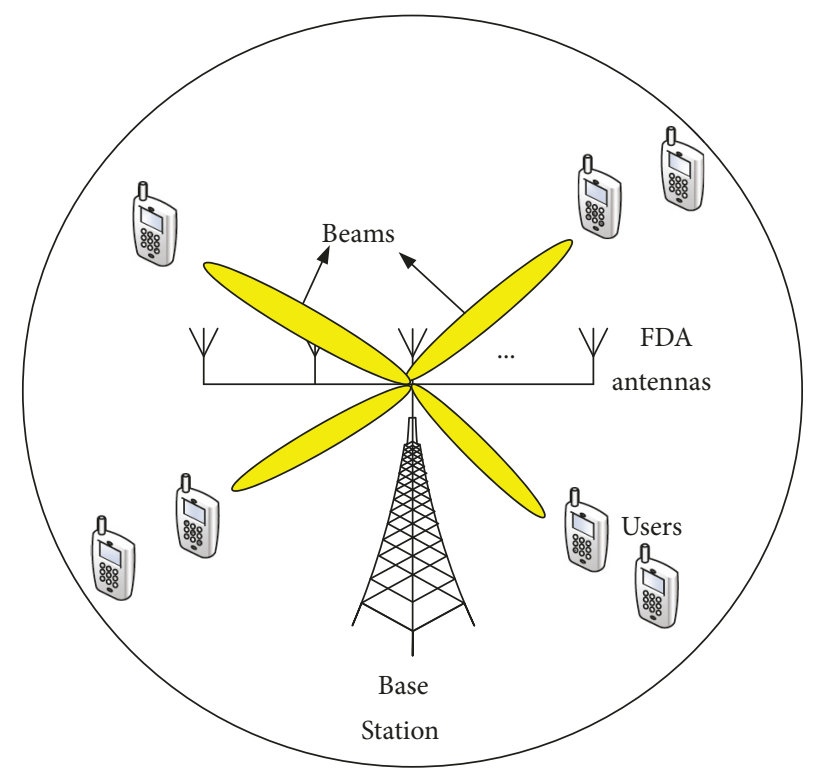

FIGURE 7: Base station FDA beamforming transmitter for millimeter-wave communications.

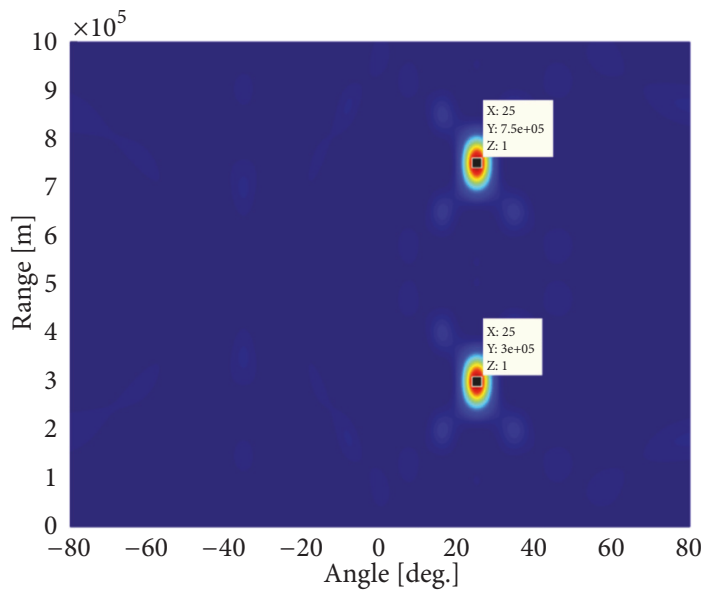

(a)

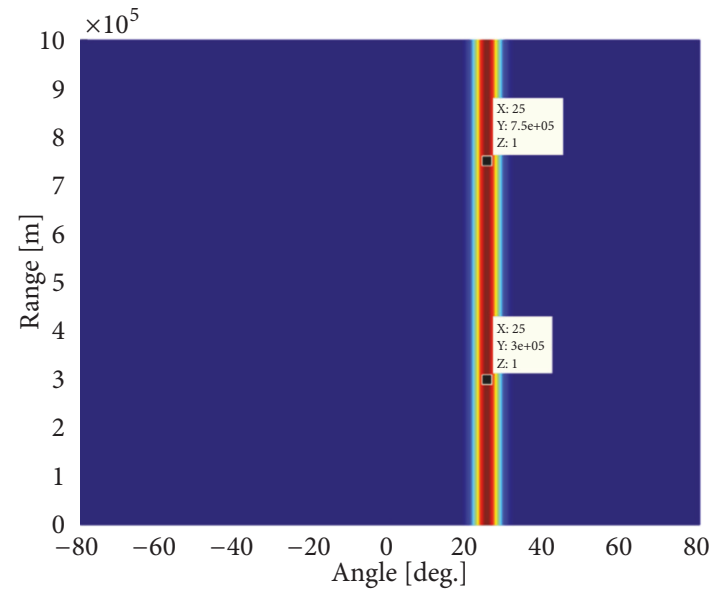

(b)

FIGURE 8: Beamforming illustration of two communication receivers: (a) FDA antenna, (b) phased-array antenna: parameters: $M=15$, $f_{0}=10 \mathrm{GHz}, \Delta f=3 \mathrm{kHz}, \varepsilon=25$.

eavesdroppers [58-62]. We briefly give two scenarios to highlight the usefulness of using FDA antennas to achieve LPI or improve security techniques in wireless communications. First, consider practical situation, where undesired receiver may be placed in the same desired direction as the desired receiver. In this case, using phased-array beamforming as shown in Figure 8(b) cannot guarantee the desired receiver secure transmission. This is because phased-array can only guarantee secure transmissions at the directions that are distinct from where the desired receiver is located. Secondly, in the case of close-located desired user and undesired user, phased-array may fail to provide better security. Therefore, in essence, FDA is a promising array technology for wireless communications, especially mm-wave $5 \mathrm{G}$ communications.
It was pointed out in [51] that FDA apparent angle $\theta_{a p}$ is different from it scanning angle $\theta$, which is

$$
\theta_{a p}=\arcsin \left(\frac{\Delta f t c}{2 \pi d f_{0}}+\sin \theta+\frac{\Delta f \sin \theta}{f_{0}}-\frac{\Delta f r}{f_{0} d}\right)
$$

This implies that (13) can be used to provide LPI characteristics as it will be difficult to predict the actual beam steering direction which is unavailable for phased-array antennas.

Next, we will analyze the bit error rate (BER) distribution of FDA in angle and range dimensions. Suppose that Gray code quadrature phase shift keyed (QPSK) symbols are adopted for transmission. Following the BER formulation in [63], we assessed the FDA BER distribution performance in Figure 9. Figure 9 illustrates FDA BER distribution in angle and range dimension, respectively. From Figures 9(a) and 


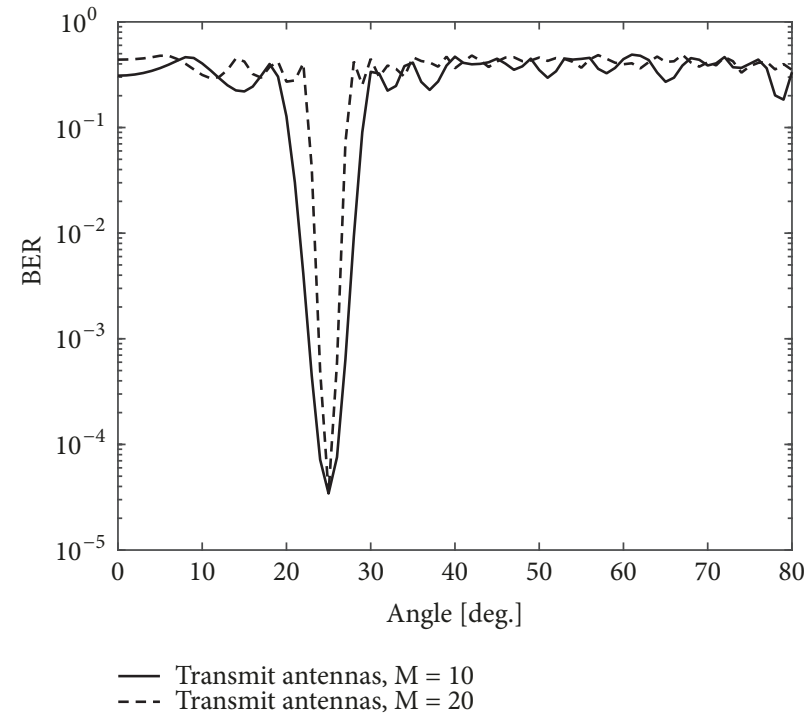

(a)

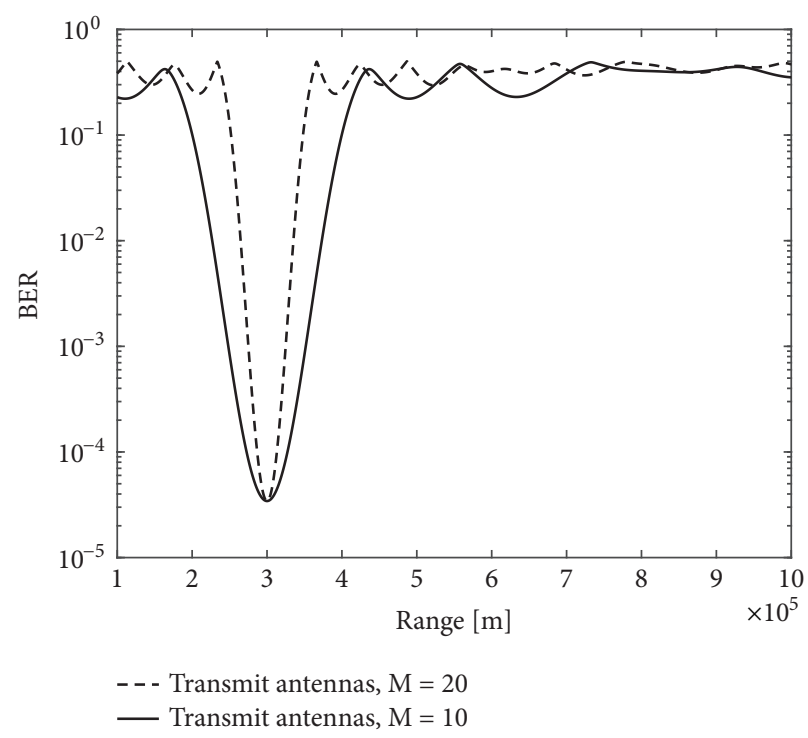

(b)

FIGURE 9: FDA BER distributions: (a) angle-dependent, (b) range-dependent: parameters: $f_{0}=10 G H z, \Delta f=3 k H z, S N R=15$.

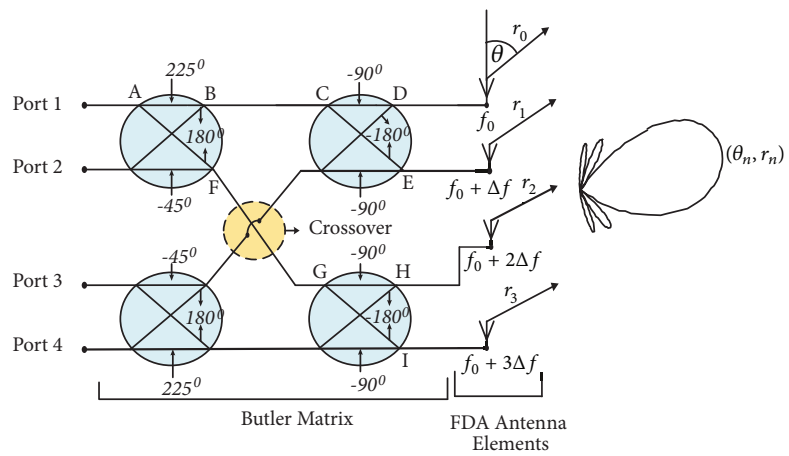

FIGURE 10: FDA $4 \times 4$ Butler matrix for multiuser mm-wave $5 \mathrm{G}$ communications.

9(b), it can be seen that as the number of transmit antennas is increasing, the BER distribution towards the intended receiver becomes narrower $\left(25^{\circ}, 300 \mathrm{~km}\right)$. It is expected that a large transmit antenna array is required to achieve narrow BER distribution for secure $\mathrm{mm}$-wave communications.

\section{FDA Array Configuration For MM-Wave Communication Applications}

4.1. FDA Butler Matrix Technique. In [64], the authors proposed FDA Butler matrix to achieve angle and rangedependent secure communications. This scheme was used to cope with the limitation of phased-array based Butler matrix in [65] which offers only angle-dependent secure communication. Figure 10 [64] shows system model of twodimensional (2D) (i.e., range and angle) half-wavelength spaced FDA antenna using a $4 \times 4$ Butler matrix. According to [65], the transfer function from the $n$th input port to the $m$ th output port of the $4 \times 4$ Butler matrix is

$$
\begin{gathered}
T_{m n}=\frac{1}{\sqrt{N}} e^{-j(2 \pi / N)(m-(N+1) / 2)(n-(N+1) / 2)}, \\
m, n=1,2, \ldots, N . \\
F_{n}(\theta, r)=\sum_{m=1}^{N}\left[\left(\frac{1}{\sqrt{N}} e^{-j(2 \pi / N)(m-(N+1) / 2)(n-(N+1) / 2)}\right)\right. \\
\left.\cdot e^{j\left[(m-(N+1) / 2) \pi \sin (\theta)-2 \pi \Delta f r / c_{0}\right]}\right]
\end{gathered}
$$

The corresponding radiation pattern at the far-field of the $n$th Butler matrix excitation input port can be expressed as in (15) [64]. As shown in (15), the main beam pointing direction depends on angle $\theta$, range $r$, and frequency increment $\Delta f$. 


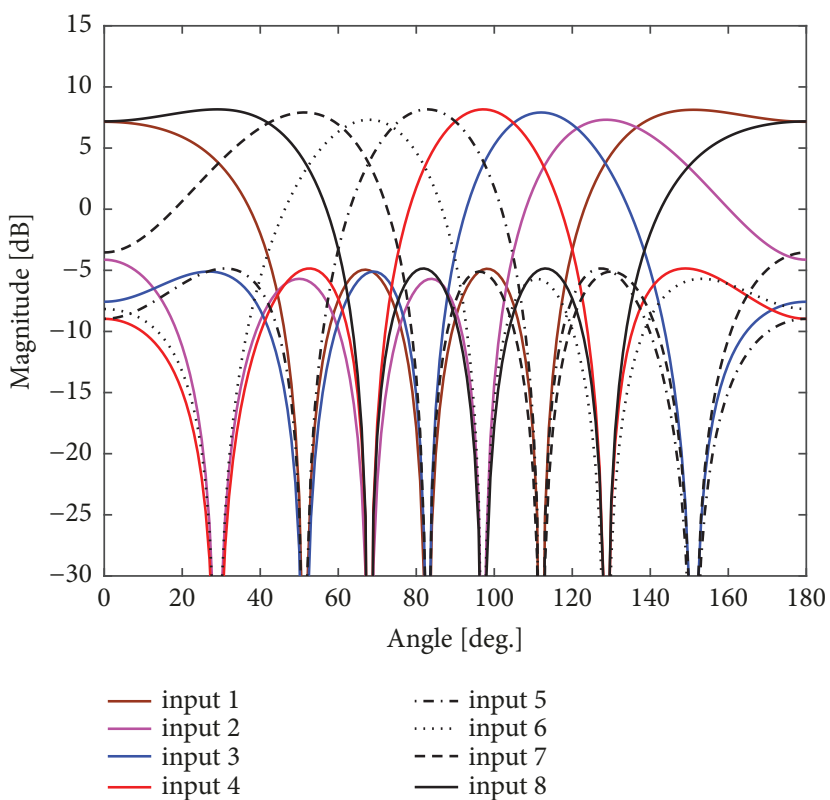

(a)

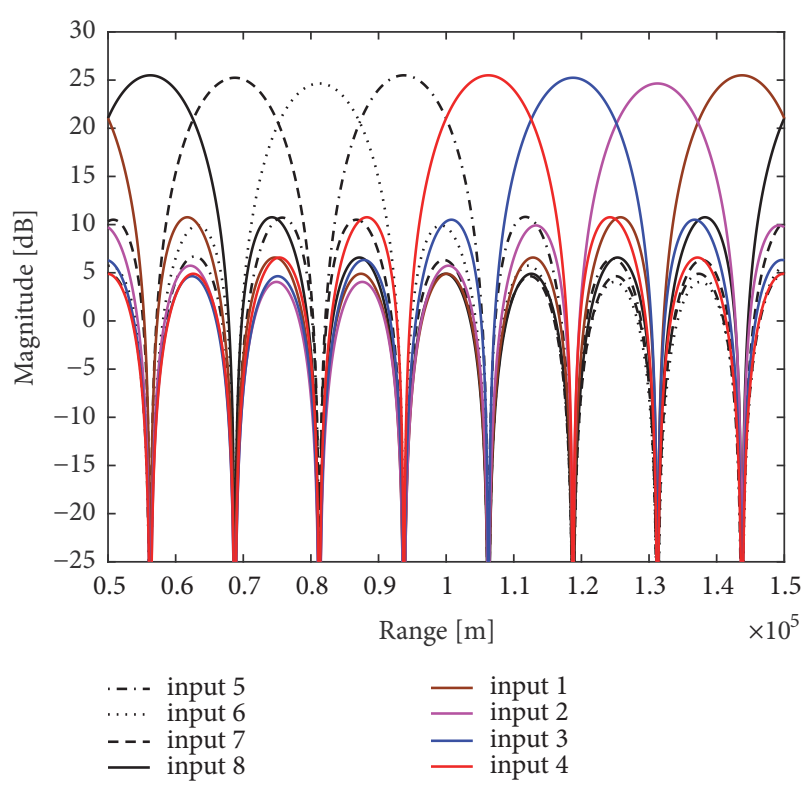

(b)

FIGURE 11: FDA $8 \times 8$ Butler matrix radiation pattern: (a) angle-dependent, (b) range-dependent: parameters: $f_{0}=10 G H z, \Delta f=100 \mathrm{kHz}$.

After some algebraic manipulations, we can obtain beampattern peak as

$$
\theta_{n}=\sin ^{-1}\left[\frac{2 n-N-1}{N}+\frac{4 \Delta f r_{n}}{c_{0}(2 m-N-1)}\right]
$$

In Figure 11, we illustrated FDA $8 \times 8$ Butler matrix using both angle focusing and range focusing (15). Hence, both angle and range dimensions can be controlled, which is beneficial for $\mathrm{mm}$-wave $5 \mathrm{G}$ communication applications.

4.2. Retrodirective Array FDA Technique. In mm-wave 5G communications, tracking of receivers at different locations is very essential. Several papers have made unrealistic assumptions that the users/receivers channel state information (CSI) is accessible at the transmitter [27, 37-40]. However, these assumptions are impractical. Besides, obtaining the users CSI at the transmitter required additional bandwidth resources. Very recently, frequency diverse phase-conjugating retrodirective array was studied in [14]. The combination of FDA and retrodirective array has unique features for $\mathrm{mm}$-wave $5 \mathrm{G}$ communications as FDA provides angle-range focusing, and retrodirective array on the other hand offers automatic tracking capability for mobile users/receivers without the need for CSI at the transmitter. Figure 12 shows the system model of frequency diverse phase-conjugating retrodirective array adopted from [14]. From Figure 12, the received signal for $m$ th element is mixed with the local oscillator (LO) signal whose frequency is twice the RF frequency plus the frequency increment, namely, $2 f_{0}+\Delta f_{m}$. Note that $f_{0} \gg \Delta f_{m}$. A clock is employed to trigger the LOs.
According to [14], the phase of the $q$ th pilot signal from an arbitrary receivers received by each element is

$$
\begin{aligned}
\psi_{q, m}(t) & =2 \pi f_{0}\left(t-\frac{r_{q, m}}{c}\right) \\
s_{o}(\theta= & \left.\theta_{q}, r, t\right) \\
= & \exp \left(j 2 \pi f_{0}\left(t-\frac{r-r^{\prime}}{c}\right)\right) \\
& \cdot \sum_{q=1}^{Q} \sum_{m=0}^{M-1} w_{m} \exp \left(j 2 \pi \Delta f_{m}\left(t-\frac{r}{c}\right)\right) \\
\times & \exp \left(j 2 \pi m d \frac{\sin \theta-\sin \theta_{q}}{\lambda}\right)
\end{aligned}
$$

where $r_{q, m}=r^{\prime}-m d \sin \theta_{q}$, with $\theta_{q}$ denoting the direction angle of incoming pilot signal from the receivers and $r^{\prime}$ is the distance from the receivers to the first element. The retransmitted signal received at the arbitrary receivers location $\left(\theta=\theta_{q}, r\right)$ is given in (18) [14]. As seen in (18), both angle and range dimensions can also be controlled, which is important for $\mathrm{mm}$-wave $5 \mathrm{G}$ communication applications.

4.3. Hybrid FDA MIMO Scheme. Large number of transmit arrays can be employed to enable different signals especially for multiple users for mm-wave communication applications. At the receiver, we can utilize matched filters to extract these signals emitted by the transmit arrays separately. Employing MIMO technology, we can achieve spatial diversity; however, it cannot offer directional beam/gain which is useful in 

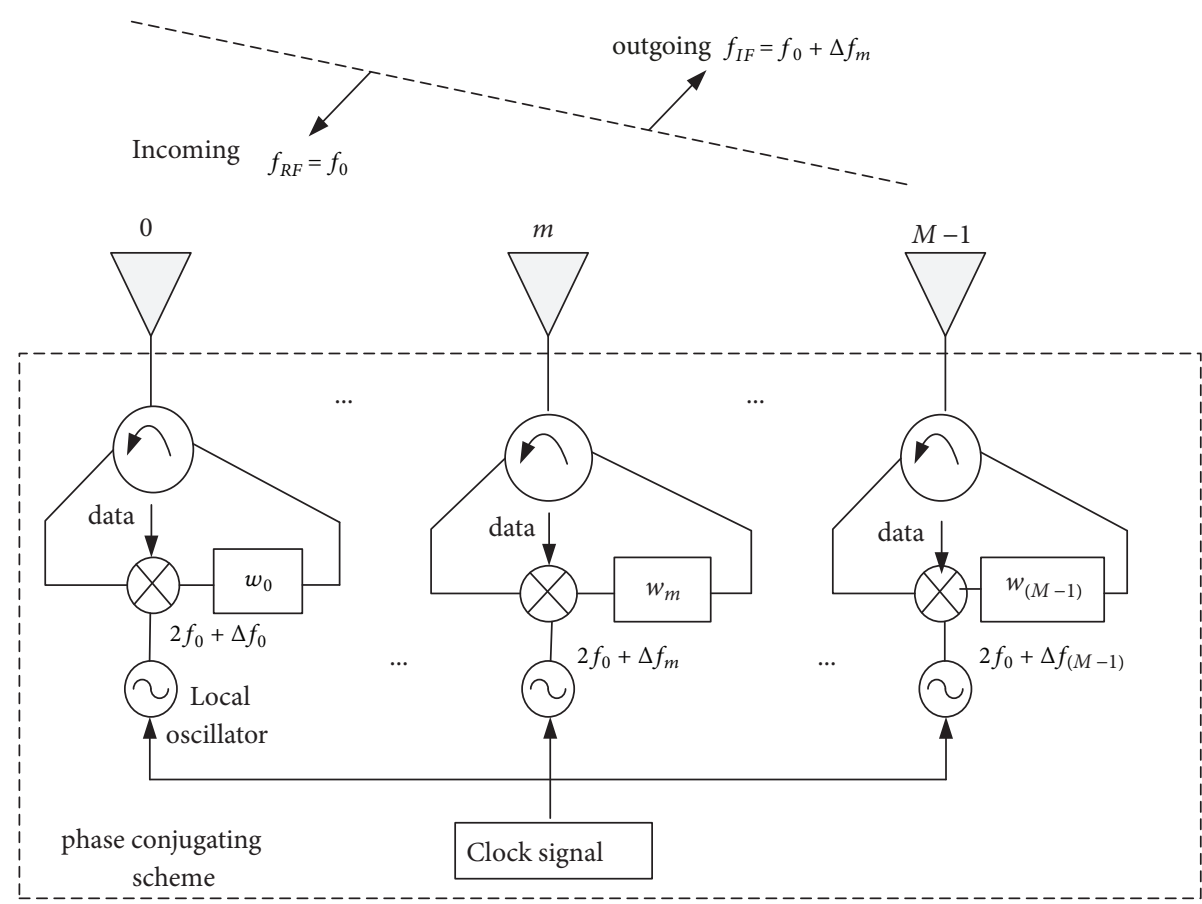

FIGURE 12: System model of frequency diverse phase-conjugating retrodirective array for mm-wave 5G communications [14].

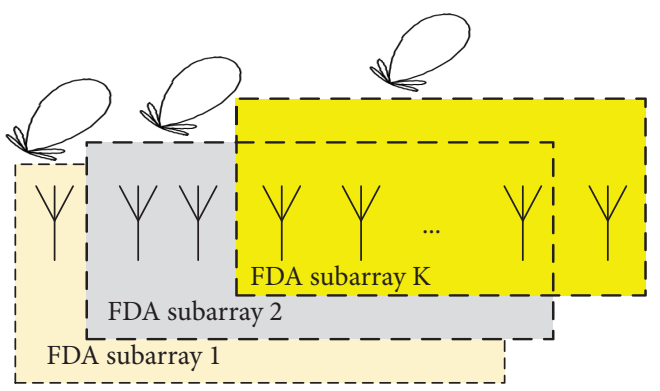

(a)

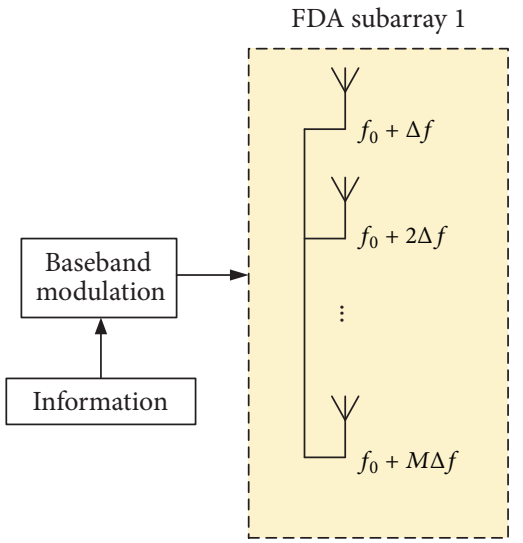

(b)

FIGURE 13: FDA MIMO configuration for multiuser mm-wave $5 \mathrm{G}$ communications.

wireless communication applications. In recent years combination of MIMO and FDA schemes has been considered for radar applications [66, 67]. In a similar fashion, it is also feasible to use FDA MIMO technique in mm-wave communications which provides more degrees of freedom than the phased-array MIMO method proposed in [68]. Figure 13 shows FDA MIMO configuration for mm-wave $5 \mathrm{G}$ communications.

From Figure 13, the hybrid FDA MIMO technique divides the transmit $M$ FDA elements into multi-subarrays (i.e., disjoint or overlapped). The baseband processor is connected to the individual subarray employing digital-to-analog converters (DACs). In the subarray, any number of transmit elements can be included and the elements in the individual subarray can transmit the same signal coherently along the desired directions in free space. Additionally, each subarray is capable of transmitting distinct signals simultaneously.

Let us assume that subarray comprising of $M_{k}=M-K+1$ is formed with $M_{k}$ less than $M$; the $K$ subarray signal model at certain time $t$ is

$$
\sqrt{\frac{E}{K}} \sum_{k=1}^{K} \mathbf{w}_{k}\left(\theta_{T_{k}}, r_{T_{k}}\right) x_{k}(t)
$$

where $x_{k}\left(\theta_{T_{k}}, r_{T_{k}}\right)$ defines the modulation transmitted signal for $k$ th subarray along the beam steering direction $\left(\theta_{T_{k}}, r_{T_{k}}\right)$; $\mathbf{w}_{k}\left(\theta_{T_{k}}, r_{T_{k}}\right)$ denote the weight beamforming vector for the active elements in a particular subarray. $\sqrt{E / K}$ provides 


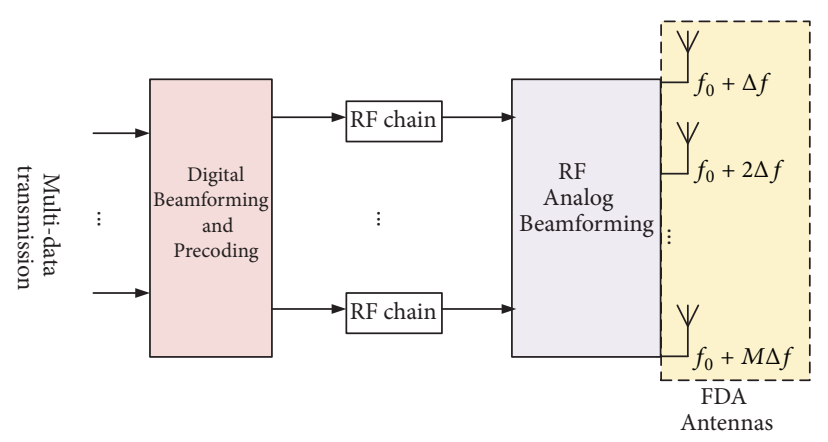

FIGURE 14: Illustration of FDA hybrid beamforming transmitter for mm-wave communication applications.

equal transmit energy for the FDA-MIMO system and $\sum x_{k}^{\dagger}(t) x_{k}(t)=1$ being the transmit energy, with ${ }^{\dagger}$ being the conjugate operation.

Without loss of generality, narrow-band channel is considered for the analysis. The signal received at time $t$ towards the location $(\theta, r)$ for any arbitrary receiver is given by

$$
\begin{aligned}
y(t, \theta, r)= & \sqrt{\frac{E}{K}} \sum_{k=1}^{K} \mathbf{h}_{k}^{H}(\theta, r) \mathbf{w}_{k}\left(\theta_{T_{k}}, r_{T_{k}}\right) \\
& \times \exp \left(-j \tau_{k}(\theta, r)\right) x_{k}(t)+n(t)
\end{aligned}
$$

where $\mathbf{h}_{k}^{H}(\theta, r)$ denotes the channel vector for the $k$ th subarray, and the conjugate transpose operator is denoted as ${ }^{H}$, $\tau_{k}(\theta, r)$ being the relative time propagation from the reference element of the first subarray, and $n(t)$ is the noise term (i.e., additive white Gaussian noise (AWGN)). Inspecting (20), it can be noticed that we can control range and angle towards the desired direction which is useful for $\mathrm{mm}$-wave communication applications.

4.4. FDA Hybrid Beamforming Transmitter Architecture for MM-Wave Communications. In mm-wave communication technology, hybrid beamforming schemes were proposed to overcome the high path loss [69-71]. Figure 14 depicts FDA hybrid beamforming transmitter for mm-wave communications. Unlike the phased-array based hybrid beamforming [69-71] with phase shifters which enable only angle beamforming with additional complexity, the FDA hybrid beamforming can enable beamforming without phase shifters in both angle focusing and range focusing. The author thinks that utilizing FDA in the hybrid beamforming techniques, many potential applications could benefit, for instance, $\mathrm{mm}$ wave outdoor communications.

\section{Optimization Technique for Maximum FDA Focusing For MM-Wave Communication Applications}

It is well known in the literature [72] that the line-of-sight (LOS) in mm-wave communications usually dominates over non-LOS so we can optimize the FDA antenna to focus the transmitted electromagnetic energy to the receiver. Note that in FDA, we can control the angle-range-time beampattern by using frequency increment and/or transmit weight vector. In doing so, the transmitted energy can be maximized to focus the gain towards the desired receiver(s).

Following the optimization problem in [73] based on discrete spheroidal sequence (DSS) [74] and without loss of generality, we consider angle-time transmit energy corresponding to the region of interest. First, the steering vector is described as

$$
\mathbf{A}(\theta, t)=\mathbf{a}_{\theta}(\theta) \otimes \mathbf{a}_{t}(t)
$$

where $\mathbf{a}(\theta, t)=\left[\begin{array}{c}\exp (j 2 \pi(\Delta f t+d \sin \theta / \lambda)) \cdots \\ \exp (j 2 \pi M(\Delta f t+d \sin \theta / \lambda))\end{array}\right]^{T}$.

The optimization problem is formulated as

$$
\begin{gathered}
\max _{\theta}\left\{\frac{\mathbf{w}_{\theta}^{H} \mathbf{A}_{\theta} \mathbf{w}_{\theta}}{2 \pi \mathbf{w}_{\theta}^{H} \mathbf{w}_{\theta}}\right\}, \quad \theta_{\min } \leq \theta \leq \theta_{\max } \\
\max _{t}\left\{\frac{\mathbf{w}_{t}^{H} \mathbf{A}_{t} \mathbf{w}_{t}}{2 \pi \mathbf{w}_{t}^{H} \mathbf{w}_{t}}\right\}, \quad t_{\min } \leq t \leq t_{\max } \\
\text { s.t } \quad \mathbf{A}_{\theta}=\int_{\theta_{\min }}^{\theta_{\max }} \mathbf{a}_{\theta}(\theta) \mathbf{a}_{\theta}^{H}(\theta) d \theta \\
\mathbf{A}_{t}=\int_{t_{\min }}^{t_{\max }} \mathbf{a}_{t}(t) \mathbf{a}_{t}^{H}(t) d t
\end{gathered}
$$

where $\mathbf{w}_{\theta}$ and $\mathbf{w}_{t}$ are represented with eigenvectors which correspond to the largest eigenvalue, namely, $\mathbf{A}_{\theta}$ and $\mathbf{A}_{t}$, respectively. Hence, using (22), we can design the transmit weight vector as $\mathbf{W}=\mathbf{w}_{\theta} \otimes \mathbf{w}_{t}$, with $\otimes$ being the Kronecker operator. In a similar manner, angle-range $(\theta, r)$ and rangetime $(r, t)$ transmit beampatterns optimization problems can be formulated for $\mathrm{mm}$-wave communication applications.

\section{Typical Fabrication/Measurement Implementation Methods}

In this section, we discuss some of the FDA fabrication methods in the literature. The authors in [75] employed waveform generator to generate certain range of frequencies. This waveform is mixed with $\Delta f$ 's (i.e., multiples). To improve the beamforming, phase control and amplitude control can be adopted at each channel. But, due to the mixers, the 


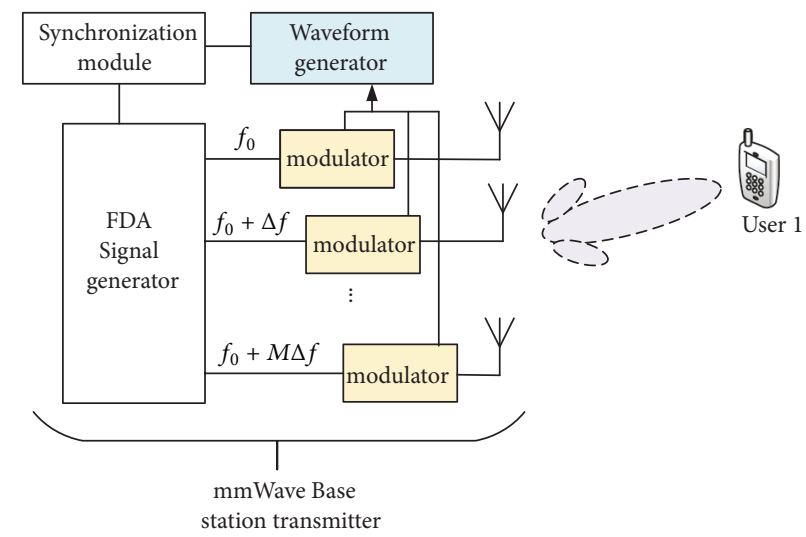

FIGURE 15: Mm-wave base station using FDA antennas for directional range-angle focusing dependent.

scheme has spectral purity challenge. Another fabrication scheme was reported in [76]. A discrete source was employed for each waveform to handle waveform diversity as well as the stepped frequency change. These devices, namely, direct digital and/or programmable phase-locked loop synthesizers can be used for the waveform generators. In [77, 78], another fabrication method was presented. In this method, the main advantage is the reliable radiation timing information due to the employment of power combiner and couplers (this is of great significance to $\mathrm{mm}$-wave communications). Note that the mixers may cause image products and intermodulation products. In [79], the author discussed another implementation method of FDA using phase locked loop (PLL) frequency synthesis techniques. The author reported that from the theoretical point of view, when the PLL reached the phase locked state, two generated signals will have identical phase and frequency $[80,81]$. Hence, we can align various PLL frequency synthesizers and then apply common reference signal, which can create the intended frequency diverse signals.

All the above mentioned fabrication schemes are suitable for mm-wave applications. In particular, generation of directional beamforming is seen as an attractive technique which can be used to alleviate high propagation path loss in mm-wave. For example, the author thinks that both the base station and communication user can support directional beamforming. The base station can adopt beamforming training to form multiple beams scanning (i.e., directional) which can be radiated simultaneously to multiple users with distinct beams within mm-wave cell. Figure 15 shows an implementation diagram of using FDA antennas at the mmwave base station for range-angle beamforming synthesis.

\section{Future Directions}

In the literature, FDA technology arrangement has been focused on monochromatic assumption with identical interelement spacing. In addition, for the sake of simplicity, several authors focused on linearly frequency increments and uniform weighting vector. Since different applications may require different techniques, the author thinks that different design approaches can be useful for particular applications. Amplitude weighting, time modulated [82, 83], and even window functions can be adopted to create different FDA beampatterns. Furthermore, since FDA antennas have inherent LPI capability, we can design LPI waveforms to provide more robust secure information transmission in wireless communication systems. FDA antennas can be regarded as a variant of MIMO, cotime, and cofrequency systems. This implies that FDA antennas can be tunable and incorporating FDA antennas into many physical hardware designs can yield extra advantages as compare to phased-array antennas.

\section{Conclusion}

This review paper discusses a new array technology, namely, FDA antenna from the perspective of narrow-band and ultrawide-band considerations. The employment of FDA technology for mm-wave communications has been discussed. Due to more degrees of freedom that can be derived from using FDA antennas, it is expected to be the most attractive antennas for wireless communication applications which has the following attributes, for example, range-dependent interferences suppression, improving beamforming gain in range-angle-time dependent which is not accessible to phased-array antennas, enhanced secure communications, better energy efficient communications, and others. Several FDA design configurations suitable for mm-wave communications have also been discussed. Further, an optimization algorithm for FDA has been highlighted which can maximize the transmitted energy in angle-time dependent. Certainly, there are more open issues in relation to the development of FDA technology for mm-wave communications. This is just a stepping stone to showcase the capability of FDA technology for $5 \mathrm{G}$ communications. Therefore, more support on FDA antennas research on $\mathrm{mm}$-wave $5 \mathrm{G}$ wireless communication systems for future is needed.

\section{Conflicts of Interest}

The author declares that there is no conflict of interest regarding the publication of this paper. 


\section{References}

[1] S. Kutty and D. Sen, "Beamforming for millimeter wave communications: an inclusive survey," IEEE Communications Surveys \& Tutorials, 2015.

[2] Y. Niu, Y. Li, D. Jin, L. Su, and A. V. Vasilakos, A Survey of Millimeter Wave (mm-Wave) Communications for 5G: Opportunities and Challenges, 2015, https://arxiv.org/abs/1502.07228? context $=$ cs.

[3] J. G. Andrews, S. Buzzi, and W. Choi, "What will 5G be?" IEEE Journal on Selected Areas in Communications, vol. 32, no. 6, pp. 1065-1082, 2014.

[4] R. Ford, M. Zhang, M. Mezzavilla, S. Dutta, S. Rangan, and M. Zorzi, "Achieving ultra-low latency in $5 \mathrm{G}$ millimeter wave cellular networks," IEEE Communications Magazine, vol. 55, no. 3, pp. 196-203, 2017.

[5] W. Roh, J.-Y. Seol, J. Park et al., "Millimeter-wave beamforming as an enabling technology for $5 \mathrm{G}$ cellular communications: theoretical feasibility and prototype results," IEEE Communications Magazine, vol. 52, no. 2, pp. 106-113, 2014.

[6] J. A. Zhang, X. Huang, V. Dyadyuk, and Y. J. Guo, "Massive hybrid antenna array for millimeter-wave cellular communications," IEEE Wireless Communications Magazine, vol. 22, no. 1, pp. 79-87, 2015.

[7] C. Sun, X. Gao, S. Jin, M. Matthaiou, Z. Ding, and C. Xiao, "Beam Division Multiple Access Transmission for Massive MIMO Communications," IEEE Transactions on Communications, vol. 63, no. 6, pp. 2170-2184, 2015.

[8] M. Xiao, S. Mumtaz, Y. M. Huang et al., "Millimeter wave communications for future mobile networks," IEEE Journal on Selected Areas in Communications, vol. 35, no. 9, pp. 1909-1935, 2017.

[9] X. Ge, R. Zi, X. Xiong, Q. Li, and L. Wang, "Millimeter Wave Communications with OAM-SM Scheme for Future Mobile Networks," IEEE Journal on Selected Areas in Communications, vol. 35, no. 9, pp. 2163-2177, 2017.

[10] L. Kong, L. Ye, F. Wu, M. Tao, G. Chen, and A. V. Vasilakos, "Autonomous Relay for Millimeter-Wave Wireless Communications," IEEE Journal on Selected Areas in Communications, vol. 35, no. 9, pp. 2127-2136, 2017.

[11] Q. Xue, X. Fang, M. Xiao, and L. Yan, "Multiuser Millimeter Wave Communications with Nonorthogonal Beams," IEEE Transactions on Vehicular Technology, vol. 66, no. 7, pp. 56755688, 2017.

[12] L. Wei, R. Hu, Y. Qian, and G. Wu, "Key elements to enable millimeter wave communications for $5 \mathrm{G}$ wireless systems," IEEE Wireless Communications Magazine, vol. 21, no. 6, pp. 136$143,2014$.

[13] W.-Q. Wang, "Ultrawideband Frequency-Diverse Array Antennas: Range-Dependent and Autoscanning Beampattern Applications," IEEE Antennas and Propagation Magazine, vol. 60, no. 3, pp. 48-56, 2018.

[14] A.-M. Yao, W. Wu, and D.-G. Fang, "Frequency diverse array phase conjugating retrodirective array with simultaneous rangefocusing capability for multitargets," in Proceedings of the AsiaPacific Microwave Conference, pp. 1-3, Nanjing, China, 2015.

[15] S. Y. Nusenu and A. Basit, "Frequency diverse array antennas: From their origin to their application in wireless communication systems," Journal of Computer Networks and Communications, vol. 2018, Article ID 5815678, pp. 1-12, 2018.
[16] P. F. McManamon, P. J. Bos, M. J. Escuti et al., "A review of phased array steering for narrow-band electrooptical systems," Proceedings of the IEEE, vol. 97, no. 6, pp. 1078-1096, 2009.

[17] J. Li and P. Stoica, "The phased array is the maximum snr active array," IEEE Signal Processing Magazine, vol. 27, no. 2, pp. 143$144,2010$.

[18] E. Brookner, "Phased array radars - Past, present and future," in Proceedings of the RADAR 2002, pp. 104-113, UK, October 2002.

[19] J. H. G. Ender, H. Wilden, U. Nickel et al., "Progress in phased-array radar applications, Microwave Symp. Digest," in Proceedings of the 2005 IEEE MTT-S International Microwave Symposium - Copyright and Reprint Permission, p. 4, 2005.

[20] N. N. Alotaibi and K. A. Hamdi, "Switched Phased-Array Transmission Architecture for Secure Millimeter-Wave Wireless Communication," IEEE Transactions on Communications, vol. 64, no. 3, pp. 1303-1312, 2016.

[21] A. H. Naqvi and S. Lim, "Review of Recent Phased Arrays for Millimeter-Wave Wireless Communication," Sensors, vol. 18, 2018.

[22] P. Antonik, An Investigation of A Frequency Diverse Array [Ph.D. thesis], University College London, London, UK, 2009.

[23] P. Antonik, M. C. Wicks, H. D. Griffiths, and C. J. Baker, "Frequency diverse array radars," in Proceedings of the IEEE Radar Conference, pp. 215-217, Verona, Italy, April 2006.

[24] W.-Q. Wang and J. Cai, "MIMO SAR using chirp diverse waveform for wide-swath remote sensing," IEEE Transactions on Aerospace \& Electronic Systems, vol. 48, no. 4, pp. 3171-3185, 2012.

[25] A. M. Haimovich, R. S. Blum, and L. J. Cimini, "MIMO radar with widely separated antennas," IEEE Signal Processing Magazine, vol. 25, no. 1, pp. 116-129, 2008.

[26] J. Li and P. Stoica, "MIMO radar with colocated antennas," IEEE Signal Processing Magazine, vol. 24, no. 5, pp. 106-114, 2007.

[27] Y. Ding, J. Zhang, and V. Fusco, "Frequency diverse array OFDM transmitter for secure wireless communication," IEEE Electronics Letters, vol. 51, no. 17, pp. 1374-1376, 2015.

[28] W. Wang, "Mitigating range ambiguities in high PRF SAR with OFDM waveform diversity," IEEE Geoscience and Remote Sensing Letters, vol. 10, no. 1, pp. 101-105, 2013.

[29] F. S. Johansson, L. G. Josefsson, and T. Lorentzon, "A Novel Frequency-Scanned Reflector Antenna," IEEE Transactions on Antennas and Propagation, vol. 37, no. 8, pp. 984-989, 1989.

[30] C. Vazquez, C. Garcia, Y. Alvarez, S. Ver-Hoeye, and F. LasHeras, "Near field characterization of an imaging system based on a frequency scanning antenna array," IEEE Transactions on Antennas and Propagation, vol. 61, no. 5, pp. 2874-2879, 2013.

[31] W.-Q. Wang, "Range-angle dependent transmit beampattern synthesis for linear frequency diverse arrays," IEEE Transactions on Antennas and Propagation, vol. 61, no. 8, pp. 4073-4081, 2014.

[32] W.-Q. Wang, "Overview of frequency diverse array in radar and navigation applications," IET Radar, Sonar \& Navigation, vol. 10, no. 6, pp. 1001-1012, 2016.

[33] H. Shao, J. Li, H. Chen, and W.-Q. Wang, "Adaptive frequency offset selection in frequency diverse array radar," IEEE Antennas and Wireless Propagation Letters, vol. 13, pp. 1405-1408, 2014.

[34] W.-Q. Wang and H. C. So, "Transmit subaperturing for range and angle estimation in frequency diverse array radar," IEEE Transactions on Signal Processing, vol. 62, no. 8, pp. 2000-2011, 2014. 
[35] Y. Wang, W. Wang, H. Chen, and H. Shao, "Optimal frequency diverse subarray design with cramér-rao lower bound minimization," IEEE Antennas and Wireless Propagation Letters, vol. 14, no. 1, pp. 1188-1191, 2015.

[36] A. Basit, W. Khan, S. Khan, and I. M. Qureshi, "Development of frequency diverse array radar technology: a review," IET Radar, Sonar \& Navigation, vol. 12, no. 2, pp. 165-175, 2018.

[37] J. Xiong, S. Y. Nusenu, and W.-Q. Wang, "Directional Modulation Using Frequency Diverse Array For Secure Communications," Wireless Personal Communications, vol. 95, no. 3, pp. 2679-2689, 2017.

[38] S. Y. Nusenu, W.-Q. Wang, and S. Ji, "Secure directional modulation using frequency diverse array antenna," in Proceedings of the IEEE Radar Conference (RadarConf), pp. 0378-0382, USA, May 2017.

[39] S. Y. Nusenu, W. Wang, and J. Xiong, "Time-modulated FDA for physical-layer security," IET Microwaves, Antennas \& Propagation, vol. 11, no. 9, pp. 1274-1279, 2017.

[40] W. Wang, "DM using FDA antenna for secure transmission," IET Microwaves, Antennas \& Propagation, vol. 11, no. 3, pp. 336345, 2017.

[41] S. Y. Nusenu and W. Q. Wang, "Range-dependent spatial modulation using frequency diverse array for ofdm wireless communications," IEEE Transactions on Vehicular Technology, vol. 67, no. 11, pp. 10886-10895, 2018.

[42] S. Y. Nusenu, S. Huaizong, P. Ye, W. Xuehan, and A. Basit, "Dual-function radar-communication system design via sidelobe manipulation based on fda butler matrix," IEEE Antennas and Wireless Propagation Letters, vol. 18, no. 3, pp. 452-456, 2019.

[43] S. Y. Nusenu and A. Basit, "Cognitive Transmit Subarray FDA Design for Integrated Radar-Communication Using Flexible Sidelobe Control," in Proceedings of the 2018 IEEE 7th International Conference on Adaptive Science \& Technology (ICAST), pp. 1-6, Accra, August 2018.

[44] S. Y. Nusenu, W.-Q. Wang, and H. Chen, "Dual-function MIMO radar-communications employing frequency-hopping chirp waveforms," Progress in Electromagnetics Research M, vol. 64, pp. 135-146, 2018.

[45] S. Y. Nusenu, W.-Q. Wang, and A. Basit, "Time-Modulated FDMIMO array for integrated radar and communication systems," IEEE Antennas and Wireless Propagation Letters, vol. 17, no. 6, pp. 1015-1019, 2018.

[46] S. Y. Nusenu and W.-Q. Wang, "Dual-function FDA MIMO radar-communications system employing costas signal waveforms," in Proceedings of the 2018 IEEE Radar Conference, pp. 33-38, USA, April 2018.

[47] W. D. Prather, C. E. Baum, R. J. Torres, F. Sabath, and D. Nitsch, "Survey of worldwide high-power wideband capabilities," IEEE Transactions on Electromagnetic Compatibility, vol. 46, no. 3, pp. 335-344, 2004.

[48] A. Dastranj, "Optimization of a Printed UWB Antenna: Application of the invasive weed optimization algorithm in antenna design.," IEEE Antennas and Propagation Magazine, vol. 59, no. 1, pp. 48-57, 2017.

[49] O. Taheri, A. Maunder, and P. Mousavi, "Correlation-Based UWB Radar for Thin Layer Resolution," IEEE Antennas and Wireless Propagation Letters, vol. 15, pp. 901-904, 2016.

[50] R. J. Fontana, "Recent system applications of short-pulse ultrawideband (UWB) technology," IEEE Transactions on Microwave Theory and Techniques, vol. 52, no. 9, pp. 2087-2104, 2004.
[51] A. Aytun, Frequency Diverse Array Radar [M.S. thesis], Naval Postgraduate School, Monterey, Calif, USA, 2010.

[52] W. Khan, I. M. Qureshi, and S. Saeed, "Frequency diverse array radar with logarithmically increasing frequency offset," IEEE Antennas and Wireless Propagation Letters, vol. 14, pp. 499-502, 2015.

[53] A. Basit, I. M. Qureshi, W. Khan, S. U. Rehman, and M. M. Khan, "Beam Pattern Synthesis for an FDA Radar with Hamming Window-Based Nonuniform Frequency Offset," IEEE Antennas and Wireless Propagation Letters, vol. 16, pp. 22832286, 2017.

[54] S. Y. Nusenu, Z. Wang, and W. Q. Wang, "FDA radar using Costas sequence modulated frequency increments," in Proceedings of the 2016 CIE International Conference on Radar (RADAR), pp. 10-13, 2016.

[55] A. Mukherjee, S. A. A. Fakoorian, J. Huang, and A. L. Swindlehurst, "Principles of physical layer security in multiuser wireless networks: A survey," IEEE Communications Surveys \& Tutorials, vol. 16, no. 3, pp. 1550-1573, 2014.

[56] A. D. Wyner, “The wire-tap channel," Bell Labs Technical Journal, vol. 54, no. 8, pp. 1355-1387, 1975.

[57] P. K. Gopala, L. Lai, and H. El Gamal, "On the secrecy capacity of fading channels," IEEE Transactions on Information Theory, vol. 54, no. 10, pp. 4687-4698, 2008.

[58] H.-M. Wang, M. Luo, Q. Yin, and X.-G. Xia, "Hybrid cooperative beamforming and jamming for physical-layer security of two-way relay networks," IEEE Transactions on Information Forensics and Security, vol. 8, no. 12, pp. 2007-2020, 2013.

[59] N. Valliappan, A. Lozano, and R. W. Heath Jr., "Antenna subset modulation for secure millimeter-wave wireless communication," IEEE Transactions on Communications, vol. 61, no. 8, pp. 3231-3245, 2013.

[60] J. Kim, A. Ikhlef, and R. Schober, "Combined relay selection and cooperative beam forming for physical layer security," Journal of Communications and Networks, vol. 14, no. 4, pp. 364-373, 2012.

[61] H. Ma and P. Ma, "Beamforming design of decode-and-forward cooperation for improving wireless physical layer security," in Proceedings of the 2013 15th International Conference on Advanced Communications Technology (ICACT), pp. 41-49, 2013.

[62] M. P. Daly and J. T. Bernhard, "Directional modulation technique for phased arrays," IEEE Transactions on Antennas and Propagation, vol. 57, no. 9, pp. 2633-2640, 2009.

[63] Y. Ding and V. Fusco, "BER-driven synthesis for directional modulation secured wireless communication," International Journal of Microwave and Wireless Technologies, vol. 6, no. 2, pp. 139-149, 2014.

[64] S. Y. Nusenu, H. Chen, W.-Q. Wang, S. Ji, and O. A. K. OpuniBoachie, "Frequency diverse array using butler matrix for secure wireless communications," Progress in Electromagnetics Research M, vol. 63, pp. 207-215, 2018.

[65] Y. Ding and V. Fusco, "Sidelobe manipulation using Butler matrix for $60 \mathrm{GHz}$ physical layer secure wireless communication," in Proceedings of the 2013 Loughborough Antennas and Propagation Conference (LAPC '13), pp. 61-65, IEEE, Loughborough, UK, November 2013.

[66] K. Gao, W. Q. Wang, and J. Cai, "Frequency diverse array and MIMO hybrid radar transmitter design via Cramér-Rao lower bound minimisation," IET Radar, Sonar \& Navigation, vol. 10, no. 9, pp. 1660-1670, 2016. 
[67] S. L. Wang, Z.-H. Xu, X. Liu, W. Dong, and G. Wang, "Subarraybased frequency diverse array for target range-angle localization with monopulse processing," IEEE Sensors Journal, vol. 18, no. 14, pp. 5937-5947, 2018.

[68] W.-Q. Wang and Z. Zheng, "Hybrid MIMO and Phased-Array Directional Modulation for Physical Layer Security in mmWave Wireless Communications," IEEE Journal on Selected Areas in Communications, vol. 36, no. 7, pp. 1383-1396, 2018.

[69] F. Sohrabi and W. Yu, "Hybrid Analog and Digital Beamforming for mmWave OFDM Large-Scale Antenna Arrays," IEEE Journal on Selected Areas in Communications, vol. 35, no. 7, pp. 14321443, 2017.

[70] S. F. Han, C.-L. I, Z. K. Xu, and C. Rowell, "Large-scale antenna systems with hybrid analog an digital beamforming for millimeter wave 5G," IEEE Transactions Communications Magazine, vol. 53, no. 1, pp. 186-194, 2015.

[71] T. E. Bogale, L. B. Le, and X. Wang, "Hybrid Analog-Digital Channel Estimation and Beamforming: Training-Throughput Tradeoff," IEEE Transactions on Communications, vol. 63, no. 12, pp. 5235-5249, 2015.

[72] Y. Ren, L. Li, G. Xie et al., "Line-of-Sight Millimeter-Wave Communications Using Orbital Angular Momentum Multiplexing Combined with Conventional Spatial Multiplexing," IEEE Transactions on Wireless Communications, vol. 16, no. 5, pp. 3151-3161, 2017.

[73] W.-Q. Wang, "Range-angle dependent transmit beampattern synthesis for linear frequency diverse arrays," IEEE Transactions on Antennas and Propagation, vol. 61, no. 8, pp. 4073-4081, 2013.

[74] P. Forster and G. Vezzosi, "Application of spheroidal sequences to array processing," in Proceedings of the IEEE International Conference on Acoustics, Speech, and Signal Processing, vol. 12, pp. 2268-2271, Dallas, TX, USA, 1987.

[75] M. C. Wicks and P. Antonik, Frequency Diverse Array with Independent Modulation of Frequency, Amplitude, and Phase, 2008.

[76] S. Huang, K. F. Tong, and C. J. Baker, "Frequency diverse array: Simulation and Design," in Proceedings of the Propagation Conference (LAPC), pp. 253-256, Loughborough, UK, November 2009.

[77] M. Secmen, S. Demir, A. Hizal, and T. Eker, "Frequency diverse array antenna with periodic time modulated pattern in range and angle," in Proceedings of the IEEE Radar Conference, pp. 427-430, Boston, Ma, USA, April 2007.

[78] T. Eker, S. Demir, and A. Hizal, "Exploitation of linear frequency modulation continuous waveform (LFMCW) for frequency diverse arrays," IEEE Trans. Antennas Propag, vol. 61, no. 7, pp. 3546-3553, 2013.

[79] J. Huang, Frequency Diversity Array: Theory and Design [Ph.D. thesis], University College London, 2010.

[80] E. R. Best, Phase Locked Loops: Theory, Design, and Applications, Mcgraw-Hill, 2007.

[81] V. F. Kroupa, Phase Lock Loops and Frequency Synthesis, 2003.

[82] W. H. Kummer, A. T. Villeneuve, T. S. Fong, and F. G. Terrio, "Ultra-low sidelobes from time-modulated arrays," IEEE Transactions on Antennas and Propagation, vol. 11, no. 6, pp. 633-639, 1963.

[83] S. Yang, Y. B. Gan, A. Qing, and P. K. Tan, "Design of a uniform amplitude time modulated linear array with optimized time sequences," IEEE Transactions on Antennas and Propagation, vol. 53, no. 7, pp. 2337-2339, 2005. 


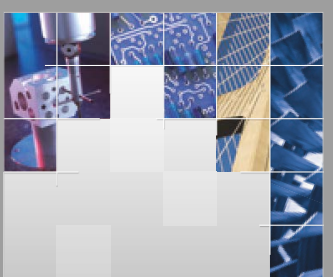

\section{Enfincering}
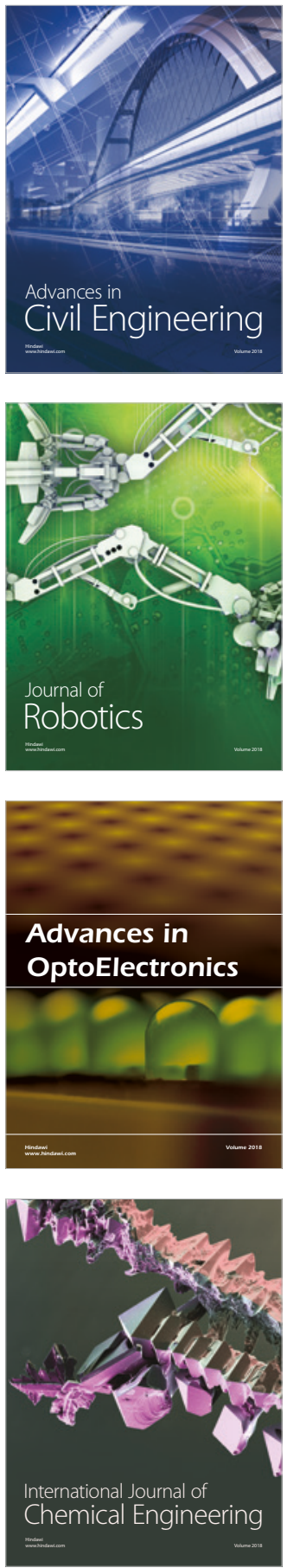

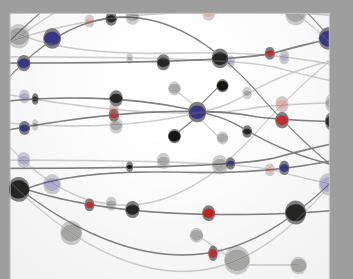

\section{Rotating \\ Machinery}

The Scientific World Journal

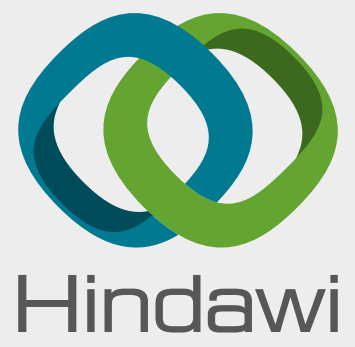

Submit your manuscripts at

www.hindawi.com
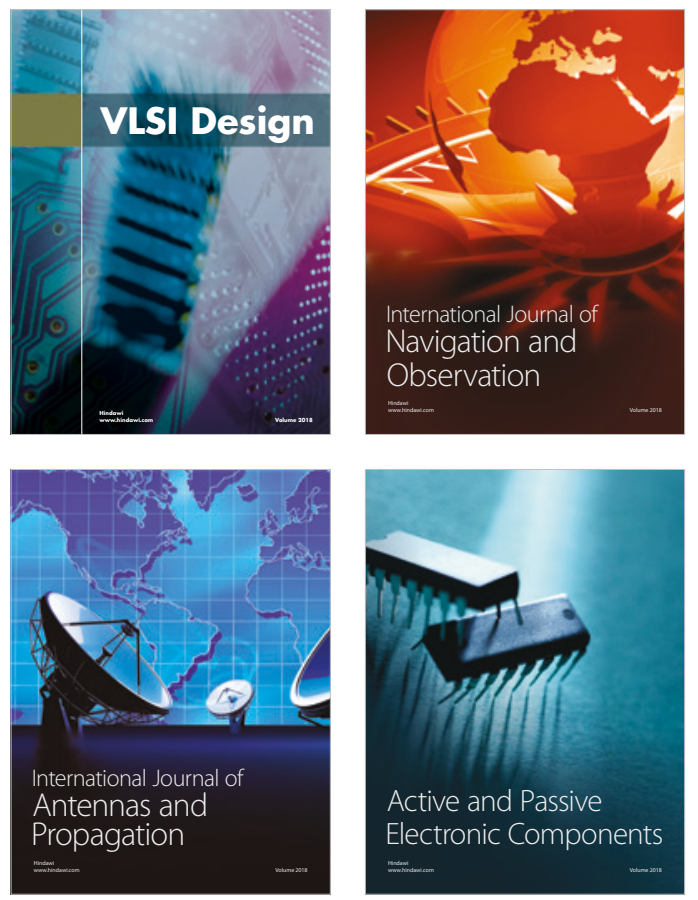
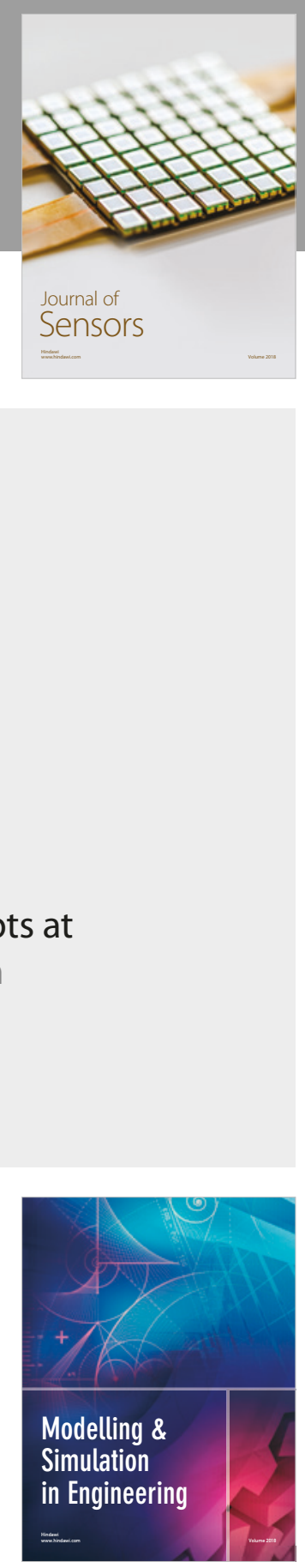

\section{Advances \\ Multimedia}
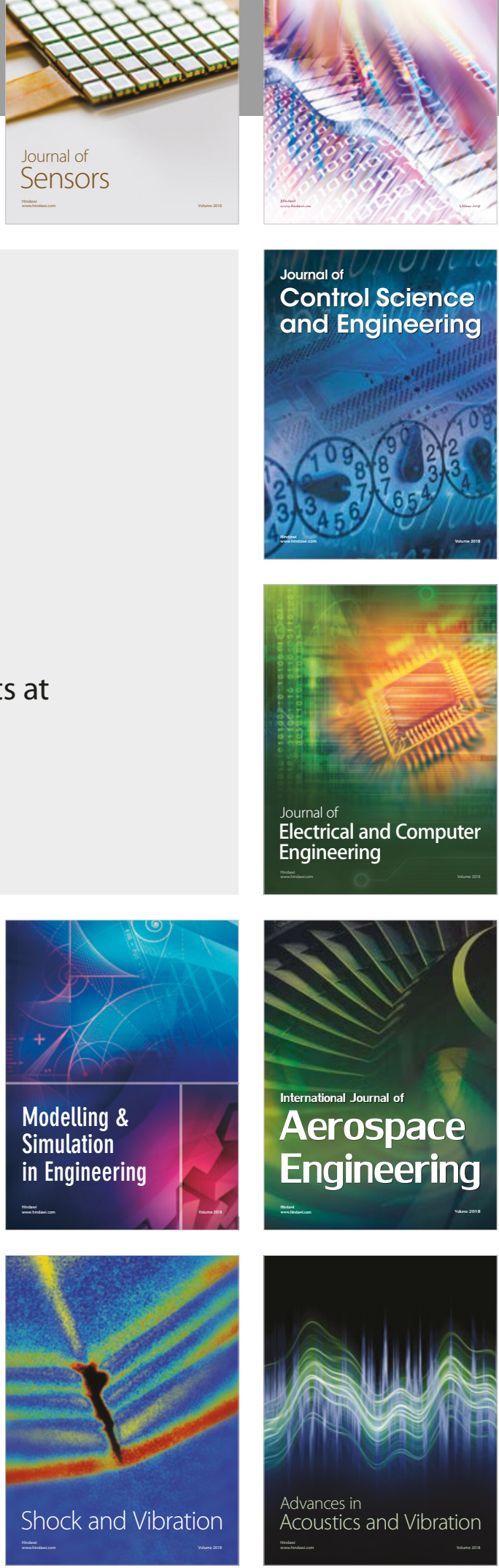